\title{
Parametric sonar for harbor and port security-two designs comparing with their linear counterparts
}

\author{
Songwen $\mathrm{Li}^{1,2}$ \\ ${ }^{1}$ Science and technology on underwater acoustic antagonizing laboratory \\ ${ }^{2}$ Shanghai marine electronic equipment research institute, 5200 Jindu road, Shanghai, 201108 \\ lisongwen@smmail.cn
}

Keywords: parametric sonar; parametric array; bottom reverberation; small objects detection

\begin{abstract}
Two designs of parametric sonar with respect to their linear counterparts which usually used as harbor and port security sonar are presented. One design is working at the frequency of 30 $\mathrm{kHz}$ according to the frequency of a linear sonar, but this frequency is the difference frequency for the parametric sonar whose primary frequency is $90 \mathrm{kHz}$. The other is working at $10 \mathrm{kHz}$ with primary frequency of $60 \mathrm{kHz}$ which is the working frequency of another linear sonar. Calculation and analyzing demonstrate that the nonlinear designs have better performance in reverberation limited environment while the linear ones work better in noise limited environment, when their apertures are the same size. This suggests that parametric sonar will have better performance at costal shallow water area where the boundary reverberation is usually the most important limiting factor for active sonar performance.
\end{abstract}

\section{Introduction}

Parametric array, with its advantage of achieving narrow beam-width with small aperture, had been widely used in underwater acoustic engineering [1][2][3]. Traditionally, the most important usages of this technology is in high resolution sub-bottom profiler [4][5]. Recently, parametric array technology has also been used as part of harbor and port security system (sea Nuvosonic) in order to achieve better sonar performance in coastal shallow water environment.

Underwater security for harbor and port is essential nowadays. In coastal shallow water environment, sonar is usually working in the reverberation background when detecting slow target such as diver and UUV. The following two designs compare the effectiveness of parametric sonar with their ordinary linear sonar counterpart working in this condition.

\section{Comparison between ordinary linear sonar and a parametric sonar with same array aperture and frequency}

a) Linear sonar.

Parameters of linear sonar:

Frequency: $30 \mathrm{kHz}$

Bandwidth: $10 \mathrm{kHz}$

Array size: $0.6 \mathrm{mx} 0.2 \mathrm{~m}$

$\theta_{-3 \mathrm{~dB}}=4.1^{\circ}$ (horizontal) and $12.3^{\circ}$ (vertical)

If the bottom backscatter coefficient for the coastal shallow water environment is $S_{b}=-35 d B$, then the reverberation level is

$$
\mathrm{RL}=\mathrm{S}_{\mathrm{b}}+10 \lg \left(\mathrm{c} \tau / 2 * \theta *_{\mathrm{r}}\right)
$$

Here $\tau=0.1 \mathrm{~ms}$ is the effective pulse width for the signal with bandwidth of $10 \mathrm{kHz}, \theta=4.1 \mathrm{o}=$ 0.07arc, $\mathrm{c}$ is sound speed and $\mathrm{r}$ is range of the target. 
If the target strength $\mathrm{TG}=-20 \mathrm{~dB}$, and the signal to noise ratio (SNR) is high enough, then the reverberation limited sonar equation is:

$$
\mathrm{DT}=\mathrm{TS}-\mathrm{RL}
$$

DT is the detection threshold. For false alarm 10-5, DT=10 [6]. Substitute equation (1) and all the parameters into equation (2), it can be solved that the detection range is

$$
\mathrm{r} \approx 600 \mathrm{~m}
$$

b) Parametric sonar

Parameters of parametric sonar

Array size: $0.6 \mathrm{~m} \times 0.1 \mathrm{~m}$

(vertical size is half of that of the linear sonar is to make the vertical beam broader in order to cover the whole depth of the coastal water, and in order to reduce the difficulties of realizing $30 \mathrm{kHz}$ difference wave, two arrays with same size are chosen)

Primary frequency f1: $75 \mathrm{kHz}$

Bandwidth of primary frequency 1: $10 \mathrm{kHz}$

Primary frequency f2: $105 \mathrm{kHz}$

Bandwidth of primary frequency 2: $10 \mathrm{kHz}$

$\theta_{-3 \mathrm{~dB}}$ (for average of the two primary frequency) $=1.4^{\circ}$ (horizontal) and $8.4^{\circ}$ (vertical)

The working frequency (difference frequency) is

$$
\mathrm{f}_{\mathrm{d}}=\mathrm{f}_{1}-\mathrm{f}_{2}=30 \mathrm{kHz}
$$

Which is the same as that of the linear sonar, and then the difference frequency is $30 \mathrm{kHz}$. The beam width of difference frequency is almost the same as that of the primary frequency, and the bandwidth of the difference frequency is $20 \mathrm{kHz}$, twice of that of primary frequency.

Apparently, since the horizontal beam width is $1 / 3$ of the linear one, and the bandwidth is three times of the linear one, for a reverberation limited environment, the sonar detection range will be 6 times the linear one, that is $3600 \mathrm{~m}$.

In fact the source level of the parametric sonar cannot support the detection range of $3600 \mathrm{~m}$. This means the parametric sonar is working in a noise limited environment instead of reverberation limited environment.

The noise limited sonar equation is:

$$
\mathrm{DT}=\mathrm{SL}-2 \mathrm{TL}+\mathrm{TS}-(\mathrm{NL}-\mathrm{DI})+\mathrm{GT}
$$

For acoustic signal at frequency of $30 \mathrm{kHz}$, the ambient noise level is $\mathrm{NL}=37 \mathrm{~dB}$ at sea level 2; double way transmission loss is

$$
2 \mathrm{TL}=2 \times(\alpha \times \mathrm{r} / 1000+20 \lg \mathrm{r})
$$

Here $\alpha$ is the absorption coefficient for the signal travels $1000 \mathrm{~m}$, which is about $5.5 \mathrm{~dB}$ for $30 \mathrm{kHz}$ signal. $\mathrm{r}$ is the target range.

Array gain $\mathrm{DI}=25.8 \mathrm{~dB}$ for the sonar working in mono-static style; time gain $\mathrm{GT}=10 \mathrm{lg} \mathrm{T}$, $\mathrm{T}$ is the pulse width.

If we choose $\mathrm{DT}=10 \mathrm{~dB}$ and $\mathrm{T}=5 \mathrm{~ms}$, for source level $\mathrm{SL}=198 \mathrm{~dB}$, the detection range is:

$$
\mathrm{r} \approx 1000 \mathrm{~m}
$$

In this parametric sonar design, the downshift ratio, which is the ratio of the average of the two primary frequency and the difference frequency, is only 3, then the transmission efficiency of 
parametric array is quite large compare to recommended ratio of about 10 by eraly researcher. About $2 \mathrm{kw}$ pulse power is needed for this design to achieve the difference source level of $198 \mathrm{~dB}$.

If the bottom backscater coefficient $\mathrm{f}$ is $\mathrm{S}_{\mathrm{b}}=-30 \mathrm{~dB}$, the detection range for the linear sonar mentioned will be reduced to around $200 \mathrm{~m}$, but will remain the same for the parametric sonar since it is still working in the noise limited condition. Otherwise, if the sea condition is at level 4, then the ambient noise level will increase $10 \mathrm{~dB}$, the detection range for the parametric sonar will be reduced to around $500 \mathrm{~m}$. It is clear that the parametric sonar, with its narrow beam width and broad bandwidth but weak source level, is more suitable in strong reverberation environment than noisy environment while linear sonar is on the contrary.

\section{Comparison between an ordinary linear sonar and a parametric sonar with same array aperture but different frequencies}

a) Linear sonar.

Array aperture: $1200 \mathrm{~mm}$

Frequency: $60 \mathrm{kHz}$

Horizontal beam width: $1.06^{\circ}$

Bandwidth: $5 \mathrm{kHz}$

According to equation (1) and (2), for $S_{b}=-30 \mathrm{~dB}$ and $T S=-20 \mathrm{~dB}$, the detection range is:

$$
\mathrm{r} \approx 550 \mathrm{~m}
$$

b) Parametric sonar
Array aperture: $1200 \mathrm{~mm}$ (using single array)
Primary frequency: $60 \mathrm{kHz}$
Prime frequency horizontal beam width: $1.06^{\circ}$
Difference frequency: $10 \mathrm{kHz}$

The beam width for the difference frequency is almost the same of that for primary frequency. When the bottom is flat, the acoustic backscatter coefficient is frequency dependent for frequency higher than $10 \mathrm{kHz}$ [6].The acoustic backscatter coefficient is increased about 3dB/octave. So, for parametric sonar working in the frequency of $10 \mathrm{kHz}$, the bottom backscatter strength is much weaker than its linear counterpart, and the sonar will be working in the noise limited condition.

Considering the noise limited condition, according to equation (5), for $\mathrm{NL}=37 \mathrm{~dB}$ and $\mathrm{TS}=-20 \mathrm{~dB}$, when the source level for the difference frequency is $190 \mathrm{~dB}$, and the pulse width is $\mathrm{T}=10 \mathrm{~ms}$ (LFM signal with bandwidth $5 \mathrm{kHz}$ ), the detection range is around $550 \mathrm{~m}$. This source level is quite easy to achieve for this parametric sonar design. If the source level is $200 \mathrm{~dB}$, then the detection range is about $1000 \mathrm{~m}$. Although the relatively low source lever will cause some difficulties for the parametric sonar to achieve longer detection range, but this kind of difficulties are relatively easer to be overcome than suppress the bottom reverberation for linear sonar. For example, the transmitted energy can be increased significantly by increasing the pulse width, which is relatively easier to realize than to increase bandwidth or decrease the beam width in order to suppress the bottom reverberation.

\section{Summary}

From comparing the two designs of parametric sonar with their linear sonar counterparts, it is clear that with its wider bandwidth or lower frequency to suppress reverberation, parametric sonar has 
much better performance in strong bottom reverberation environment, which is coincident with almost all the harbor and port underwater environment. Thus, parametric sonar technology is recommended for the harbor and port underwater security.

\section{Reference}

[1] N. Jacobsen, G. Sundin and J. Pihl, System for mono- and bi-static sonar investigation of buried objects, Proceedings of Oceans Europe 2005, IEEE, 1147-1150

[2] R. Galvin and L. S. Wang, Measured channel characteristics and the corresponding performance of an underwater acoustic communication system using parametric transduction, IEE Proc.-Rudul: Sonar. Nuvig.. 2000l. 147, 247-253

[3] M. E. Zakharia, C. Pollet and E. Regaud, Combined parametric synthetic and interfrometric sonar for the detection of buried objects, Proceedings of Oceans Europe 2005, IEEE, 522-526

[4] B Woodward, J C Cook, A D Goodson and P A Lepper., A PHASE STEERED PARAMETRIC ARRAY FOR SUB-BOTTOM PROFILING, Proceedings of Electronic Engineering in Oceanography, Conference Publication No 394, IEEE 1994, 77-82

[5] A. Caiti, O. Bergem and J. Dybedal, Parametric sonars for seafloor characterization, Meas. Sci. Technol. 1999, 10, 1105-1115

[6] A. D. Waite, Sonar for Practising Engineers, John Wiley \& Sons, Ltd, 2002. 